\title{
Kidney Tumour Segmentation
}

\author{
A \\ Manuscript \\ submitted in partial fulfilment of \\ the \\ KiTS Challenge 2019 \\ Organised by \\ University of Minnesota \& University of Melbourne \\ by \\ Mr. Rochan Sharma \\ Data Scientist, Strategy and Architecture, Centre of Excellence \\ Reliance Industries Limited
}

Under the guidance:

Mr. Kiran Chaudhari

AVP, Strategy and Architecture, Centre of Excellence

Reliance Industries Limited 


\section{KiTS Challenge 2019}

\section{ABSTRACT}

Medical Image Segmentation is a challenging field in the area of Computer Vision. In this work Two deep learning models were explored namely U-Net and ENet. The reason to shortlist U-Net was it is suitable on a small data set and also originally designed for Biomedical Image segmentation. However when compared to ENet it is much slower. To speed up the process of Kidney Tumor segmentation , ENet was shortlisted and also experimented on the data set provided. ENet was very fast as compared to U-Net, However some visual representations of the predicted results have shown promising results in U-Net better then ENet.

A classification model called as Xception Model was also considered right in the first phase, so as to shortlist those slices from the CT which have the presence of Kidney. So that the output obtained can be given as an input to semantic segmentation model. This would allows us to speed up the process of Kidney Tumor Segmentation.

Finally performance parameter which was used for evaluation segmentation models was IOU. 


\section{KiTS Challenge 2019}

\section{Acknowledgement}

I wish to express my sincere thanks to Mr. Kiran Chaudhari, Assistant Vice President, Reliance Industries Limited for his guidance and giving me the opportunity in participating in KiTS challenge 2019 and providing me with all the necessary facilities for this research work.

I am grateful to Dr. Pallavi Halarnkar, Lead Data Science at Centre of Excellence, Reliance India Limited, as well as, all the team members of Data science for extending their continuous help in completing this work. Their expertise and valuable guidance has motivated me in completing this work.

I am also thankful to my parents for the unceasing encouragement, support and blessings they have been providing me throughout.

I would also express my sense of gratitude to one and all, who directly or indirectly have helped in one way or the other. 


\section{TABLE OF CONTENTS}

Sr. No

Topic

Page No

1. Introduction

1.1 Overview

1

1.2 About KiTS challenge 2019

1.3 Problem Statement 3

$\begin{array}{lll}1.4 & \text { KiTS dataset description } & 4\end{array}$

$\begin{array}{lll}1.5 & \text { Hardware Specifications } & 4\end{array}$

2

Literature Review

3 Implementation Methodology

3.1 Overview of the Proposed Methodology 7

3.2 U-Net Semantic Segmentation Model 8

3.3 A Real time Semantic Segmentation Model : 10 ENet 


\section{KiTS Challenge 2019}

\section{CHAPTER 1}

INTRODUCTION

This section gives and overview of Medical Image segmentation and its importance

\subsection{Overview}

Image segmentation is a very important step in a number of medical imaging studies. This process can be facilitated by Automatic Computational Techniques.

Medical Image segmentation is the process of detecting boundaries within the given $2 \mathrm{D}$ or $3 \mathrm{D}$ images collected from medical imaging devices. Some of the most important aspects in clinical oncology includes detection, localization, diagnosis, staging and monitoring treatment. In this, early detection, localization and staging can improve the survival and management in patients prior to the decided treatment therapy. Therefore it is important that collaborating techniques of Computer Vision in medical imaging localization could help assist doctors in identifying the malignant area in the given images.

\subsection{About KiTS Challenge 2019}

KiTS is organized by University of Minnesota and University of Melbourne, supported by Climb 4 Kidney Cancer (C4KC) and National Cancer Institute, of the National Institutes of Health. Ground truth labels have been provided for each patient who undergo partial or radical nephrectomy. A total of 210 out of 300 cases have been released for model training and validation, and the remaining 90 will be used for model evaluation.

In Kidney cancer, kidney cells become malignant (cancerous) and develop into a tumor. Practically all kidney tumors initially look like tiny tubes (tubules) inside the Kidney. Some doctors also call this cancer as renal cell carcinoma.

Because of the wide assortment in kidney and kidney tumor morphology, there is at present extraordinary eagerness in studying how tumor morphology can be identified within results which can help in planning treatment. 


\section{KiTS Challenge 2019}

Using the current state of art in Deep Learning, one of the approach which can be used to address this can be semantic segmentation using various deep learning models which can help overcome morphological heterogeneity problem.

Fortunately a large portion of kidney malignant growths are found before they spread (metastasize) to far off organs. Tumors are simpler to treat effectively if they are detected in early stage. However, these tumors can develop enormously into a large size before they are identified.

kidney malignant growth is seen in individuals older than 40 years of age [1]. Below are some factors responsible for kidney malignant growth:

1. Smoking: It has been seen that kidney malignant growth is twice in smokers when compared to non-smokers.

2. Being male: Males are twice as likely to get kidney malignant growth when compared to females

3. Overweight: Additional body weight can result in hormonal changes which in turn increases the risk factor.

4. Consumption of certain drugs: Non recommended drugs can also increase the risk

5. Propelled Kidney infection : Long time exposure to Dialysis

6. Genetic Condition: Individuals having hereditary papillary renal cell carcinoma or von Hippel-Lindau (VHL) infection

7. Family History: Having a family ancestry of kidney disease. Siblings have particularly high risk of these type of risk.

8. Exposed to harmful chemicals: Being presented to specific synthetic concoctions, for example, asbestos, cadmium, benzene, natural solvents, or certain herbicides.

Kidney is an essential and very important part of human body and needs to work properly otherwise human being can be severely affected. 


\section{KiTS Challenge 2019}

Unfortunately in Kidney Tumor, knowing where cancer is present in a precise manner is very difficult, it leads to potentially dangerous situations for patients too. Professional medical imaging still requires expert human interpretation for cancer detection in the kidney. This leads to very subjective, highly variable renderings.

Detection of such a severe disease is very important for all the human being because of its adverse effect, also the treatment is too costly that.

Doctors are able to diagnose it when patient approaches to them, but it will be better if the disease can be predicted much more in advance. Patients will be benefited if the detection can be done at an early stage that will help in early treatment.

\subsection{Problem Statement}

To build a Model for Tumor segmentation in Kidney that will help medical experts to have a support system that can automatically and accurately segment tumor in kidney, if a kidney is having malignant cell presence.

\subsection{KiTS Dataset Description}

\subsubsection{Data Format}

The imaging and its corresponding ground truth labels were furnished in an anonymized NIFTI Format with shape (num_slices, height, width). Here, num_slices denotes the axial view and a progress from superior to inferior as the slice index increases. In all cases, the patient was lying while image gathering, and the height-width origins along these lines lie to the patients' left front anteriors. At the point when there were multiple qualifying series for a particular case, that with the smaller slice thickness was picked, yet slices' thicknesses range from $1 \mathrm{~mm}$ to $5 \mathrm{~mm}[2]$.

\subsubsection{Ground Truth Labels}

Manual segmentation labels were done under the supervision of clinical chair, Dr. Christopher Weight [2]. 


\section{KiTS Challenge 2019}

Annotators approached each case's going according to radiologist's decision, also the conclusion is drawn from careful pathology, which encouraged for precised location of tumors and also helps in excluding of cysts. All cases were then looked into in both the axial and coronal planes. Rectifications were made by Nicholas Heller under the directions of Drs. Christopher Weight and Niranjan Sathianathen where every needed.

\subsection{Hardware Specification}

To train and test the Deep Learning Models, following are the details of a cloud GPU used for carrying out the implementation and testing.

Operating System: Windows Server 2012R2

GPU Type and Size: Standard NV12 (12 VCPUS, 112 GB RAM), 2 core GPU with 8 GB RAM 


\section{KiTS Challenge 2019}

\section{CHAPTER 2}

\section{LITERATURE REVIEW}

The current state of art has many techniques implemented for detection of kidney tumor, a few of them have been mentioned below.

Qian Yu et al. [3] proposed a CNN model, Crossbar-Net, which comprises of two primary developments. One is the utilization of crossbar patches, which spread over the kidney tumor in both vertical and horizontal directions and catch the nearby and relevant data at the same time. The other one is a cascaded boosting straining style with a cover re-sampling system. In Crossbar-Net, the result of segmentation of one sub-model can be supplemented by tuning the other submodel, and each sub-model can perform better by doing re-sampling on the missegmented locale. The blend of the spread re-sampling technique and basic sampling strategy not just improves in regions that are mis-segmented, yet in addition improves sub-models from being over-emphasis learning on the missegmented areas. The model, at the same time can gain information from the variety of data and accomplish promising division results on various size, shape, different type of kidney lumps present in kidney.

Anis Ahmad et al. [4] exhibits the accurate segmentation of the tumor from the Kidney using free hand 2D ultrasound. The unguided approach used accomplish supreme results because of more images that are obtained throughout the phantom, the width of the phantom was $3 \mathrm{~cm}$ which is still clinically important. Extra manual editing has been done for obtaining more accuracy.

Mostafa Atri et al. [6] observed at tumor evaluation utilizing single planes versus multiple plane, breath hold versus free breathing and large region of interest versus focusing on the region of most elevated vascularity.

Aicha Belgherbi et al. [7] tried a semi-automatic technique for the segmentation of kidneys. The procedure utilized depends on numerical morphology. The calculation that was executed is tried on 21 CT pictures got from various patients. When it is assessed on the 21 image database, the proposed calculation resulted in great outcomes and exhibitions acquiring an affectability of $95 \%$ and a particularity of $99 \%$. This technique was originally developed for kidney 


\section{KiTS Challenge 2019}

segmentation, but can also be used for liver segmentation and lesion detection in liver or other organs, if the organ is having some kind of abnormalities present in it.

Chernoff et al. [8] developed three-dimensional (3D) pictures of renal tumors utilizing contrast enhanced spiral computed tomography (CT) to characterize the tumor's area and relationship to the kidney surface. This 3D spiral CT can help in the arranging of partial nephrectomy and in accomplishing total resection of renal cell carcinoma while saving the normal healthy renal tissue.

Remi Cuingnet et al. [9] introduced a completely programmed technique to segment and detect both kidneys in any CT volume utilizing classification forests and random regression. Regression forests were utilized to get the kidneys positions. A classification forest was used to acquire a likelihood map of kidneys. After that segmentation was done with a template deformation algorithm. Results demonstrate that the technique gives a precise of $80 \%$ in segmentation of the cases.

Hanford J. Deglint [10] has performed the segmentation of medical images for a few type of tumors. The algorithm used are 3D fuzzy connectivity algorithm and relaxing grid parameters for segmentation algorithm. The 3D connectivity algorithm was satisfactory on the tumors that are highly homogeneous, but it performed poorly on heterogeneous tumors.

Guanyu YANG [11] presented an automated multi-atlas kidney segmentation. In the first step, down-sampled patient image is matched with a set of low-resolution atlas images, and in the second step the kidney is cropped from original images and aligned with another set of high-resolution atlas images separately to calculate the fine results. The average Dice similarity coefficient and surface-tosurface distance between segmentation results and reference standard are 0.952 and $0.913 \mathrm{~mm}$. 


\section{KiTS Challenge 2019}

\section{CHAPTER 3}

\section{PROPOSED METHODOLOGY}

\subsection{Overview of Proposed Methodology}

Computer Vision is an interdisciplinary field that deals with how computer systems can be made to gain useful knowledge from images and videos. It automates the task of Human Visual System (HVS).

Two such tasks includes, Image classification and Semantic Segmentation. The solution proposed includes the following two phases.

1. Image classification (Presence of Kidney)

2. Semantic Segmentation (Kidney and Tumor segmentation)

\section{Phase 1: Classification}

The proposed solution includes the first phase, where a deep learning model called as Xception model is trained for classification of slices for the presence of Kidney. This approach helps in saving computational time complexity of semantic segmentation approach. The first phase of classification helps in shortlisting the ROI (Region of Interest), the slices containing presence of Kidney, so that only those slices having the presence of kidney with respect to a patient are given to a semantic segmentation model from finding the presence of Tumor.

Different classification Models like VGG-16, VGG-19 and Inception Deep learning models were also experimented for classification, however Exception models turned out to be better than the remaining ones.

\section{Phase 2: Semantic Segmentation}

In this phase the output obtained from a classification model (shortlisted slices) are now given as an input to a Semantic segmentation Model for detecting kidney tumor. Two deep learning Models were experimented, U-Net and ENet.

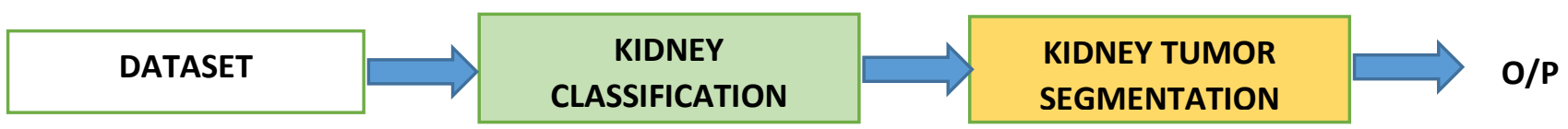

Fig. 3.1 Block Diagram of Approach used for Kidney Tumor Segmentation 


\section{KiTS Challenge 2019}

\subsection{U-Net Semantic Segmentation Model}

Although there are lots of architecture available in deep learning for the segmentation purpose, but U-Net was developed for segmentation in Biomedical images. U-Net Model has the ability to learn from a very small dataset of like 30-35 images [5].

The U-Net architecture achieves very good performance on different biomedical segmentation applications. It only needs very few annotated images and has a very reasonable training time to learn segmentation. Thus, U-Net Model was shortlisted for KiTS challenge 2019.

The U-Net [5] architecture is based upon the Fully Convolutional Network and adjusted such that it can perform better segmentation in the medical imaging as in medical imaging very less amount of the labeled training data. Compared with FCN-8, the two principle that are used in the u-net are:

1. Architecture of $U$-net is full symmetric.

2. The skip connections between the upsampling path and the downsampling path apply a concatenation administrator rather than an entirety.

The skip associations mean to give local data to the global data while upsampling. On account of its symmetry, the system has an enormous number of feature maps in the upsampling way, which allows to exchange of information. The basic FCN architecture in its upsampling way thus had number of classes in features maps [12].

The system architecture is represented in Figure 3.2. It comprises of a contracting path (left side) and a expansive way (right side). The contracting path pursues the layers of a convolutional stacked one after other followed by max-pool. It comprises of the repeated utilization of two $3 \times 3$ convolutions (unpadded), each pursued by an rectified linear unit (ReLU) and a 2x2 max pooling activity with stride of 2 in down sampling [5]. 


\section{KiTS Challenge 2019}

At each downsampling step the number of highlight channels get doubled pursued by a $2 \times 2$ convolution ("up-convolution") that divide the feature maps in half , a concatenation with the correspondingly divide the feature maps from contracting path, and two $3 \times 3$ convolutions, each pursued by a ReLU [5].

The cropping is important because of the loss of pixels on the border in each convolution. At the last layer a $1 \times 1$ convolution is utilized for the mapping of 64feature vector to the desired number of classes. Altogether the system has 23 convolution layers [5]. The Unet model have the conv2d block that will use convolutional layers, followed by Batch normalization and the Relu activation function.

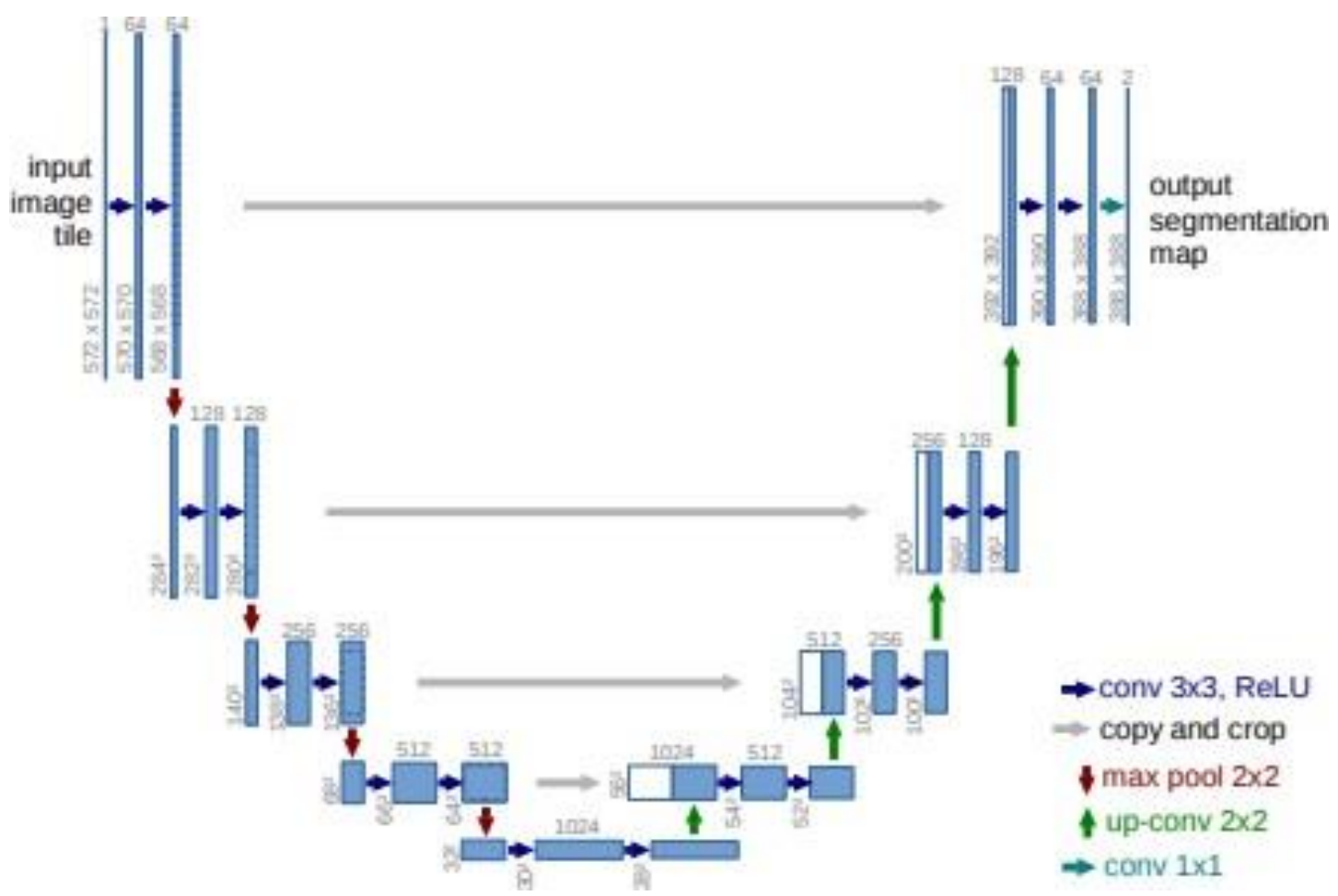

Figure 3.2: U-net architecture, each blue box represents a multiple channel feature map. On top of the each box, number of channels is denoted. The arrows are denoting the different types of operations [5]. 


\section{KiTS Challenge 2019}

\subsection{A Real time Semantic Segmentation Model : ENet}

ENet ( Efficient Neural Network) is used for pixel wise semantic segmentation. ENet is upto $18 x$ faster, requires $75 x$ less FLOPs, has $79 x$ less parameters and provides similar or better accuracy when compared to existing models.

One of the main reason why ENet was shortlisted as another model for experimenting Kidney Tumor detection was its speed. The ENet Architecture is shown below in Figure 3.3.

\begin{tabular}{lcc}
\hline Name & Type & Output size \\
\hline initial & & $16 \times 256 \times 256$ \\
\hline bottleneck1.0 & downsampling & $64 \times 128 \times 128$ \\
$4 \times$ bottleneck1.x & & $64 \times 128 \times 128$ \\
\hline bottleneck2.0 & downsampling & $128 \times 64 \times 64$ \\
bottleneck2.1 & dilated 2 & $128 \times 64 \times 64$ \\
bottleneck2.2 & asymmetric 5 & $128 \times 64 \times 64 \times 64$ \\
bottleneck2.3 & dilated 4 & $128 \times 64 \times 64$ \\
bottleneck2.4 & dilated 8 & $128 \times 64 \times 64$ \\
bottleneck2.5 & asymmetric 5 & $128 \times 64 \times 64 \times 64$ \\
bottleneck2.6 & dilated 16 & $128 \times 64 \times 64$ \\
bottleneck2.7 & upsampling & $64 \times 128 \times 128$ \\
bottleneck2.8 & & $64 \times 128 \times 128$ \\
\hline Repeat section 2, without bottleneck2.0 & $64 \times 128 \times 128$ \\
\hline bottleneck4.0 & ups \\
bottleneck4.1 & & $16 \times 256 \times 256$ \\
bottleneck4.2 & & $C \times 512 \times 512$ \\
\hline bottleneck5.0 & upsampling & $16 \times 256 \times 256$ \\
bottleneck5.1 & &
\end{tabular}

Figure 3.3 ENet Architecture (o/p sizes are given for 512×512 input) [13] 


\section{KiTS Challenge 2019}

\section{CHAPTER 4}

\section{EXPERIMENTAL RESULTS}

For Experimental results, the U-Net model was trained on 200 Patients data and 10 Patients data was reserved for validation. Finally the Model was tested for 90 cases where no ground truth was available. The obtained results are given in the following section.

IOU (Intersection over Union) is the performance parameter which is used for evaluation of the Models.

\subsection{U-Net Results}

\section{Case 1: U-Net Model on 10 cases reserved for Validation}

(a) ORIGINAL IMAGE

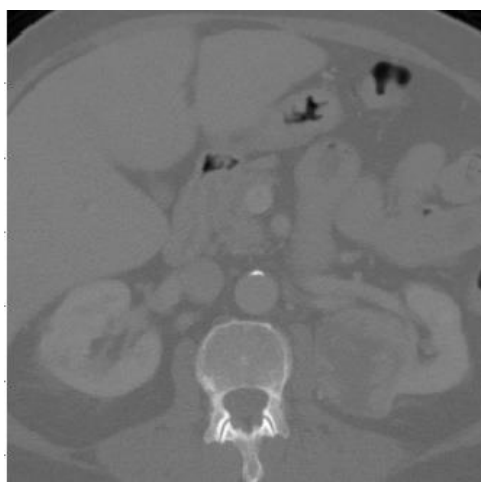

(a) ORIGINAL IMAGE
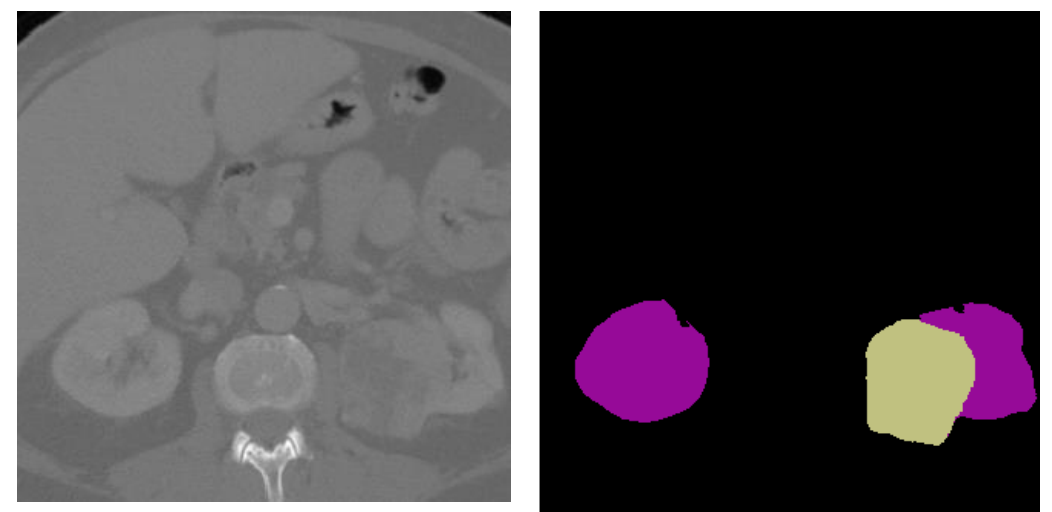

(b) GROUND TRUTH

(c) PREDICTION
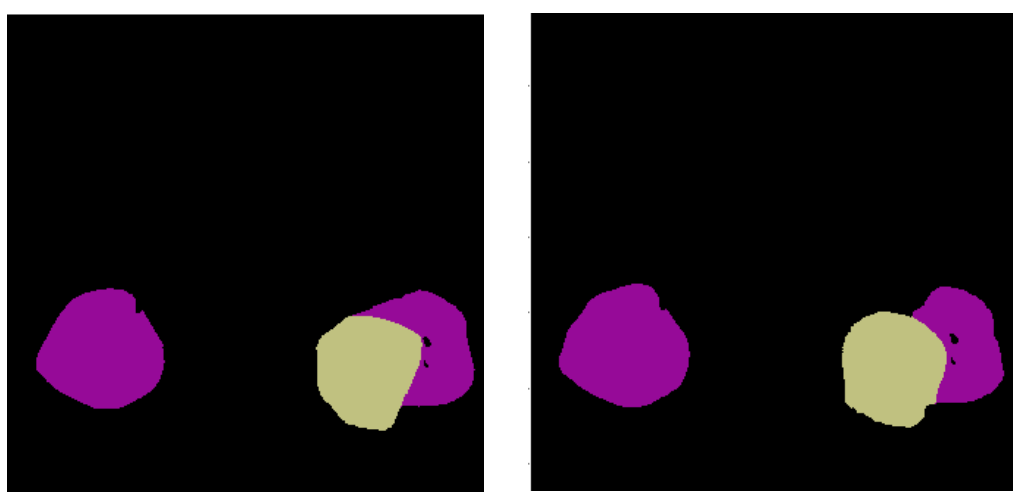

(c) PREDICTION

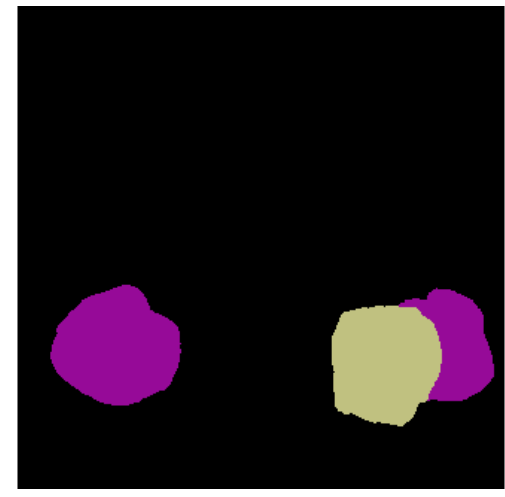

Figure 4.1. Sample Images showing (a) original image (b) Ground truth (c) Prediction by ENet Model 


\section{KiTS Challenge 2019}

In case 1, a total of 475 slices were tested it gave a value of IOU Kidney 0.8154 and IOU Cancer 0.9128

Case 2: U-Net on 90 cases where ground truth was not available

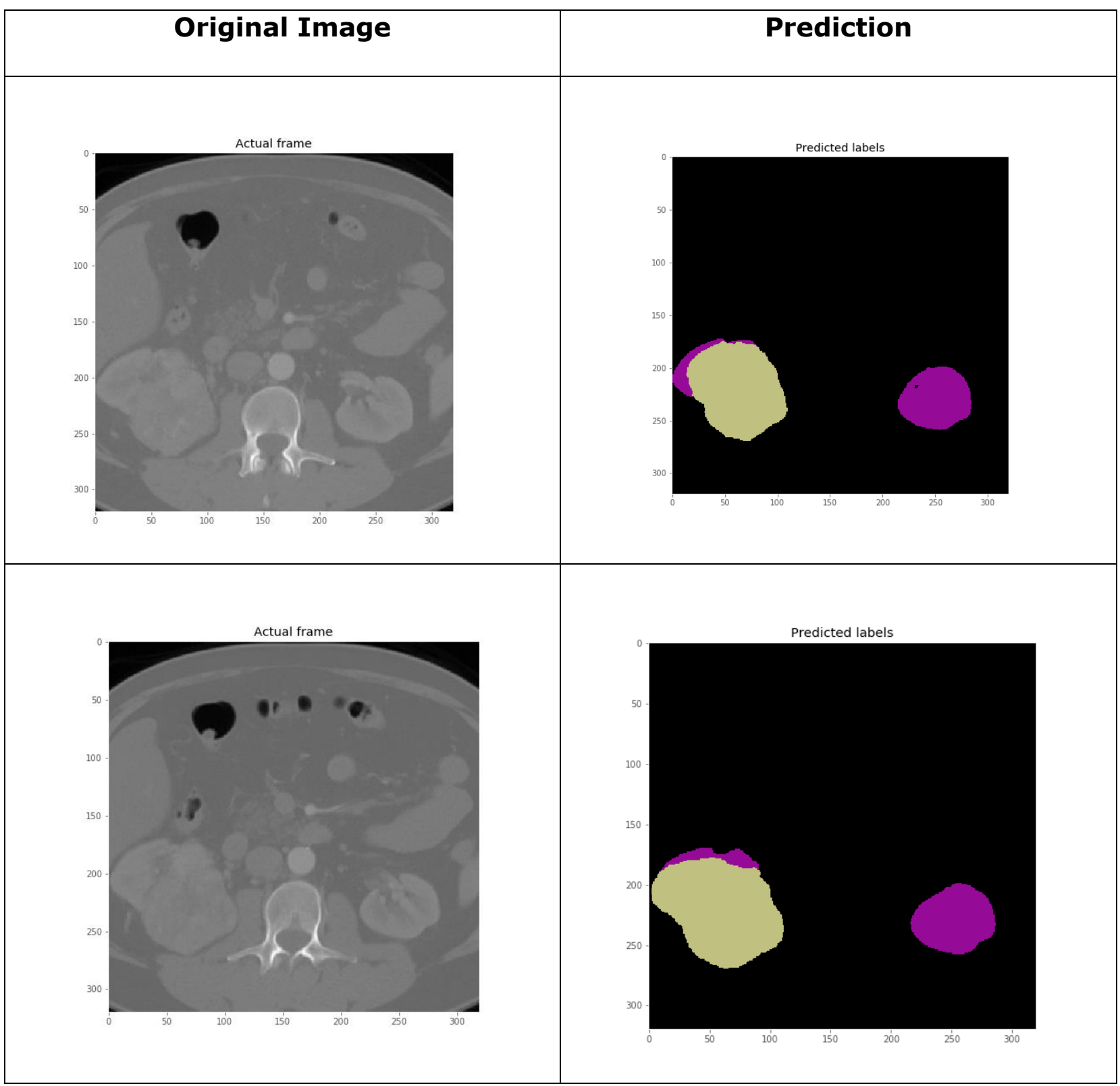




\subsection{ENet Results}

\section{KiTS Challenge 2019}

For Experimental results, the ENet model was trained on 13,415 images and validated on 1578 and tested on 790 images. Finally the Model was tested for 90 cases where no ground truth was available. The obtained results are given in the following section.

IOU (Intersection over Union) is the performance parameter which is used for evaluation of the Models.

Case 3: ENet Model on 10 cases reserved for Validation

(d) ORIGINAL IMAGE

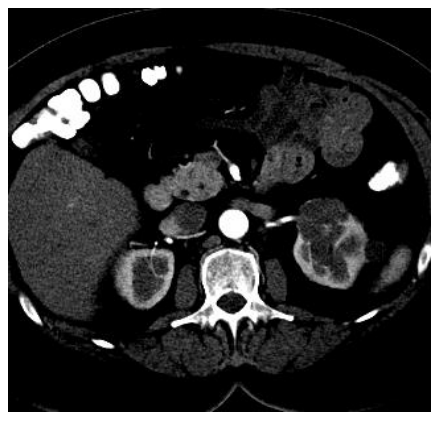

(d) ORIGINAL IMAGE

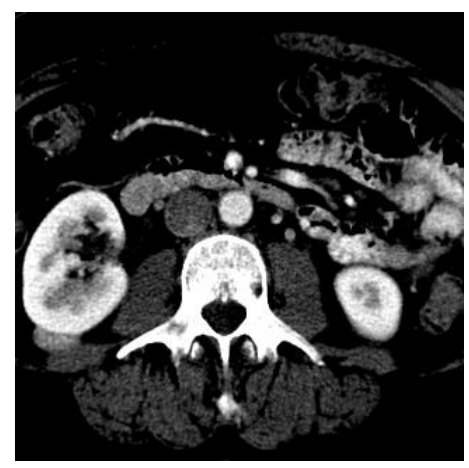

(e) GROUND TRUTH

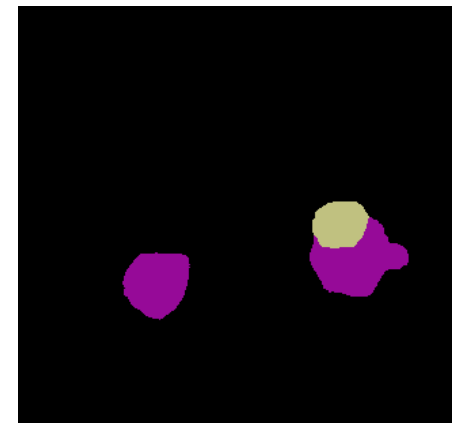

(e) GROUND TRUTH

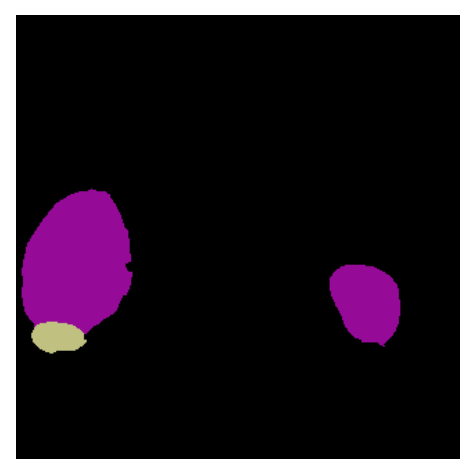

(f) PREDICTION

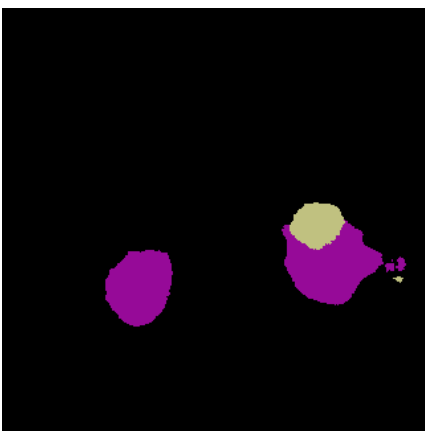

(f) PREDICTION

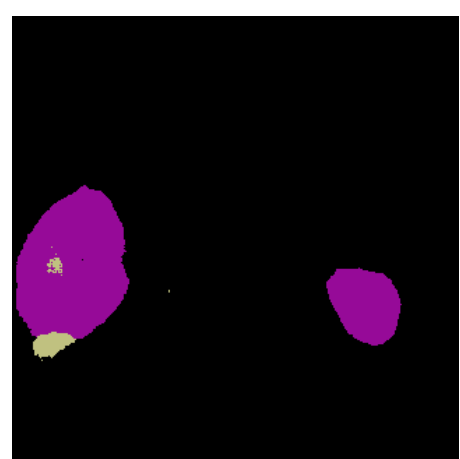

Figure 4.3. Sample Images showing (a) original image (b) Ground truth (c) Prediction by ENet Model

Note: It has been observed that when ENet was tested on 790 slices, the Model gave an IOU for Kidney as 0.8 and Tumor as 0.3 , Hence the model was not evaluated on Test images (90 cases where ground truth is not available) 


\section{KiTS Challenge 2019}

\section{CHAPTER 5}

\section{CONCLUSION AND FUTURE WORK}

Kidney Tumor Segmentation was tried using two Deep learning Models. The main Model focused was U-Net model and for improving speed E-Net model was used. The performance parameter used for testing deep learning models was IOU.

The U-Net model was trained on 27 Epochs and ENet was trained on 500 Epochs. It has been seen in the experimental results that when U-Net was test on 90 cases where ground truth was not available and the same has been uploaded on the required portal

Hence it can be concluded that U-Net is better than E-Net

In future, other deep learning models can be explored for semantic segmentation. And the these models can trained for more number of epochs 


\section{KiTS Challenge 2019}

\section{REFERENCES}

[1]. Nicholas Heller, Panagiotis Stanitsas, Vassilios Morellas, and Nikolaos Papanikolopoulos. A web-based platform for distributed annotation of computerized tomography scans. In Intravascular Imaging and Computer Assisted Stenting, and Large-Scale Annotation of Biomedical Data and Expert Label Synthesis, pages 136145. Springer, 2017.

[2]. Nicholas Heller, Niranjan Sathianathen, Arveen Kalapara, Edward Walczak, Keenan Moore, Heather Kaluzniak, Joel Rosenberg, Paul Blake, Zachary Rengel, Makinna Oestreich, et al. The kits19 challenge data: 300 kidney tumor cases with clinical context, ct semantic segmentations, and surgical outcomes. arXiv preprint arXiv:1904.00445, 2019.

[3]. Qian Yu, Yinhuan Shi, Jinquan Sun, Yang Gao, Yakang Dai, and Jianbing Zhu. Crossbar-net: A novel convolutional network for kidney tumor segmentation in ct images. arXiv preprint arXiv:1804.10484, 2018.

[4]. Anis Ahmad, Derek Cool, Ben H Chew, Stephen E Pautler, and Terry M Peters. 3d segmentation of kidney tumors from freehand $2 \mathrm{~d}$ ultrasound. In Medical Imaging 2006: Visualization, Image-Guided Procedures, and Display, volume 6141, page 61410S. International Society for Optics and Photonics, 2006.

[5]. Olaf Ronneberger, Philipp Fischer, and Thomas Brox. U-net: Convolutional networks for biomedical image segmentation. In International Conference on Medical image computing and computer-assisted intervention, pages 234-241. Springer, 2015.

[6]. Mostafa Atri, John M Hudson, Mehrdad Sinaei, Ross Williams, Laurent Milot, Hadas Moshonov, Peter N Burns, and Georg A Bjarnason. Impact of acquisition method and region of interest placement on inter-observer agreement and mea- surement of tumor response to targeted therapy using dynamic contrast-enhanced ultrasound. Ultrasound in medicine \& biology, 42(3):763-768, 2016.

[7]. Aicha Belgherbi, Ismahen Hadjidj, and Abdelhafid Bessaid. Morphological segmentation of the kidneys from abdominal ct images. Journal of Mechanics in Medicine and Biology, 14(05):1450073, 2014.

[8]. Daniel M Chernoff, Stuart G Silverman, Ron Kikinis, Douglass E Adams, Steven E Seltzer, Jerome E Richie, and Kevin R Loughlin. Three-dimensional imaging and displayof renal tumors using spiral ct a potential aid to partial nephrectomy. Urology, 43(1):125-129, 1994.

[9]. Rémi Cuingnet, Raphael Prevost, David Lesage, Laurent D Cohen, Benoît Mory, and Roberto Ardon. Automatic detection and segmentation of kidneys in $3 \mathrm{~d}$ ct images using random forests. In International Conference on Medical Image Computing and Computer-Assisted Intervention, pages 66-74. Springer, 2012.

[10]. Hanford J Deglint, Rangaraj M Rangayyan, Fábio J Ayres, Graham S Boag, and Marcelo K Zuffo. Three-dimensional segmentation of the tumor in computed tomographic images of neuroblastoma. Journal of digital imaging, 20(1):72-87, 2007. 


\section{KiTS Challenge 2019}

[11]. Guanyu Yang, Jinjin Gu, Yang Chen, Wangyan Liu, Lijun Tang, Huazhong Shu, and Christine Toumoulin. Automatic kidney segmentation in ct images based on multiatlas image registration. In 2014 36th Annual International Conference of the IEEE Engineering in Medicine and Biology Society, pages 5538-5541. IEEE, 2014.

[12]. http://www.deeplearning.net/tutorial/unet.html

[13]. Adam Paszke, Abhishek Chaurasia, Sangpil Kim, Eugenio Culurciello, "ENet: A Deep Neural Network Architecture for Real-Time Semantic Segmentation", June 2016, arXiv: 1606.02147 